\title{
Architectural Composition: A Systematic Method to Define a List of Visual Attributes
}

\author{
Seyed Farhad Tayyebi, Yüksel Demir \\ Istanbul Technical University, Istanbul, Turkey \\ Email: tayyebi.sf@gmail.com
}

How to cite this paper: Tayyebi, S. F., \& Demir, Y. (2019). Architectural Composition: A Systematic Method to Define a List of Visual Attributes. Art and Design Review, 7, 131-144.

https://doi.org/10.4236/adr.2019.73012

Received: May 7, 2019

Accepted: June 8, 2019

Published: June 11, 2019

Copyright $\odot 2019$ by author(s) and Scientific Research Publishing Inc. This work is licensed under the Creative Commons Attribution International License (CC BY 4.0).

http://creativecommons.org/licenses/by/4.0/

\section{(c) () Open Access}

\begin{abstract}
Architects are now capable to construct diverse architectural compositions with various formal attributes. Although theorists have defined diverse sets of composition attributes, no former list covers the features of our newly built buildings. This study, for the first time, introduces a systematic method to define all the visual attributes in the composition of a building. Arising from the definition of composition, the proposed method, after defining the composition layers and families, prepares a composition graph; then, by introducing three roots of the attributes, it creates a list of the visual attributes. To give a better insight, the method applied to four buildings and their visual attributes are extracted accordingly. The employment of the procedure on a set of building images sharing a criterion can reflect the most in-common formal attributes among them. Therefore, a list of building attributes are also prepared by applying the method on 200 randomly selected building images. The proposed method is adjustable to our needs and also applicable to various art forms which the term composition covers. Consequently, the introduced method has the potential to be an assistive tool in many formal explorations studies.
\end{abstract}

\section{Keywords}

Architectural Composition, Composition Syntax, Formal Attributes, Visual Attributes

\section{Introduction}

Composition has experienced multiple definitions over its centuries of background. Alberti defines composition as "the procedure in painting whereby the parts are composed together in the picture" (Grayson \& Alberti, 1972: p. 73). In architecture, Gaudet defines composition as "the combination of parts in a co- 
herent whole" (Lucan, 2012: p. 158). Jon Brantingham, the composer of Hollywood, defines musical composition as "the process of making or forming a piece of music by combining the parts, or elements of music" (Brantingham, 2019). They generally emphasize the syntax of composition, which mostly concerns the orders and arrangements of the parts. From this perspective, a decent composition requires syntactical features. Despite considering various essential traits, the initial feature, as well as the final aim of composition, is mentioned deeply but concisely as "the achievement of unity" (Lucan, 2012: p. 235). As Blanc, Pontremoli, and Dews put it, the parts of good compositions are unified in such a way that any changes would not make it better, if not destroy it (Dews, 2003; Lucan, 2012: p. 24). From this viewpoint, composition is profoundly defined as "variety in unity"; "seeking variations within unification and seeking unity within varieties" (Li, 2010). From another point of view, some theorists concern the semantic aspect of composition; they mostly concern the perception of a composition and focus more on content and artistic communication by visual elements (Aldrich, 1969). Accordingly, the trace of sign and symbols emerges in a composition (Frutiger, 1989; Langer, 1957), rather than the arrangement and unification of the physical elements. This paper, without concerning the semantic aspects of composition, concerns the syntactic aspect of composition focusing on the physical elements and their attributes.

Syntactically, the composition elements and principles are the basis of the composition attributes. As they are analogous to noun and verb in a design language (Kasprisin, 2011), Dew expresses that most of the artists' decisions are "based on the elements and principles of design" (Dews, 2003: p. 13). Composition elements and composition parts are generally used interchangeably in literature, and they refer to the composition constituents. As the word part may recall, what to assume as a composition influences on parts recognition. For example, Alberti defines "parts of a 'historia' [painting] are the bodies, parts of the bodies are member, and part of member is the surface" (Grayson \& Alberti, 1972: p. 73). In an architectural plan as a composition, the rooms would be its parts; and for a building façade, the windows, roofs, railings and so on are their composition parts (Kruger, 1991); what to consider as a composition defines a proper list of its elements.

As the second subject, composition principles involve the rules and methods of organizing the parts. Although Greg Albert discusses various composition principles based on his unique rule "never make any two intervals the same" (Albert, 2003), some theorists provide a list of shared techniques and rules, including graduation, hierarchy, contrast, complexity, contradiction, balance, and so on (Meiss, 2013). Diverse literatures reflect some architecture composition principles, which are mostly explored by building analysis via diagrams and abstract sketches (Clark \& Pause, 2012; Krier, 2010). Among them, some theorists and researchers like Hanlon and Pend believe in existing timeless composition principles (Hanlon, 2009; Li, 2010), while others believe in periodical maxims 
and formulas which are fluctuating over the ages. Gargus defines a set of fundamental transformations of forms by some principles and asserts that "the emphasis on specific principles can shift" over the time (Gargus, 1994). The exaggerated verticality of Middle Ages has been substituted with horizontality and moderated proportion through Renaissance (Arnheim, 2009). The symmetrical axis-based architecture of the 19th century has shifted toward equilibrium and balance until the late 19th century, then inclined toward the unbalanced and asymmetrical plan (Lucan, 2012: p. 221). Then, Le Corbusier defines a modular system to unify the composition elements (Corbusier, 1954) and, recently, Schumacher discusses the ontological shift from the ideal rigid geometrical figure with straight-line toward the dynamic and adaptive geometrical entities via spline and nurbs (Schumacher, 2015: p. 11). Despite various epochal shifts, in practice, some theorists like Leon Krier and Andres Duany "follow any pre-conceived set of design principles"; others like Ron Kasprisin's believes on the combination of various principles (Barnett, 2013), reminding Eclecticism in art. Consequently, regardless of the different perspectives and various fluctuations, some believe that the "formal rules derived from the academies" must be eliminated to promote "the personal inventiveness" (Caniggia \& Maffei, 2001: p. 31 ). From this perspective, Wright in the $20^{\text {th }}$ century says, "Composition [as a method] in architecture is, I hope, dead" (Wright, 1928: p. 259); lastly, the term "Non-composition" emerges to escape the compositional modes and move beyond the composition principles (Lucan, 2012).

In recent decades, architects radically questioned the sufficiency of the composition principles, and rejected any restriction by the former governing rules of composition. Nowadays, architects move beyond the limitation of the rules and technological advancements eliminate the limitation of the composition elements. Thus, new forms have been emerged in architecture, even in wooden structure buildings (Fallacara, Pantaleo, \& Scaltrito, 2019). Tokajuk by comparing the building forms during three decades reflect the evolution line of the architectural forms in Poland (Tokajuk, 2019). As Breen discuss the phenomena of architectural composition (Breen, 2019), many scholars via different perspective reflect the emergence of formal revolutions and some search for an appropriate design pattern (Jiang \& Qian, 2019). There is no constraint in either composition elements or the composition principles; accordingly, very diverse composition forms and visual attributes exist in our buildings. Even the unique formal attributes changed into the remarkable signature of the building's architect. Various formal features in the building composition are considered as by architects. We can find the importance of shadow by the platonic elements in Lois Kahn's building, significance of proportion in Le Corbusier's work, massive elements in the majestic compositions of Mario Botta, smoothness in Greg Lynn and Zaha Hadid's work, whiteness in the Richard Meier's buildings, and extreme complexity within Frank Gehry's work, the variety of artistic textures in Jean Nouvel's buildings, and straightforward design in the minimalist architectures of 
Peter Zumthor.

Nowadays, any formal attributes are visible in recent buildings. As the manipulation of the attributes and visual properties can radically influence on building forms, identifying and perception of the formal attributes are emphasized by many theorists, practitioners, and teachers. Although various set of composition attributes can be found in literature, they do not cover all the existing ones in our diverse buildings forms; lack of a proper method for defining a comprehensive list resulted in introducing multiple sets of attributes prepared by the subjective opinion of the theorists. Accordingly, this study aims to introduce a systematic method to extract the composition attributes of a building, to be further progressed to a comprehensive list of composition attribute. To prepare the method, at first, the composition attributes are extracted from both discursive theories and building forms in practice, via analyzing over 150 building images. Several classifications have explored their basis. Then, a systematic procedure is created to reveal the roots of the attributes in layer-base conforming the essence of composition, leading to a comprehensive list of the attributes. Finally, although the method is proposed in a simple manner, it went a long way to design a valid systematic method to cover all the visual attributes of a building composition. The procedure has two phases; at first, a composition graph with its components is prepared; afterward, the graph will lead us to define a proper list of composition attributes in a more objective manner. Consequently, after discussing the procedure in the next part, it is applied on four buildings to reveal their composition attributes and illustrate the method in practice.

\section{Defining Attribute Procedure}

The procedure aims to introduce a systematic method for defining the composition attributes of a building. The proposed method concentrates on building façades, though it can disclose the composition attributes of different objects which the term composition covers. Generally, regardless of the composition principles, the composition elements and the composed object as physical elements are the main roots of the attributes. Thus, at first, the objects and its composition elements are identified, to prepare the composition graph of the building. Finally, after defining their properties, a proper list of composition attributes will be extracted.

\subsection{Composition Layers and Scales}

Regarding the definition of composition, composition elements are assembled or organized to form a composed object, known as a unified object. That is, a unified object (a composition) contains various elements (parts) connected together, each can be allocated to a different layer of composition. From the scale point of view, the elements belong to a smaller scale, and the unified object is located in a larger scale. Thus, each composition has 2 main layers, the layer of the elements in a smaller scale and the layer of the unified object on a larger scale. Ac- 
cordingly, a composition layer refers to a specific scale in which the composition elements or the unified object of a composition exist within. Figure 1 illustrates the definition of composition and its assigned composition layers.

Each composition element might be considered as a unified object of other smaller-scaled elements. Likewise, a unified object can also be a component of another larger-scaled composition. Composition has chain-like composition elements and unified object, possessing a fractal essence (Figure 2, right). On one side, any architecture element is composed by some material formed by some ingredients. On the other side, as Durand discusses "Buildings are the elements of which cities are composed" (Durand, 2000: p. 143); Blondel similarly believes on "no discontinuity between architectural and urban design" (Lucan, 2012: p. 17). From this perspective, the chain of composition can exceed the architectural realm, from material science to urban studies. To focus on architectural composition attributes, the fractal nature of composition needs a proper limitation for both the smallest and the largest scales (Figure 2, left).

Among the concentrated scale range, there might be a various number of intermediate layers. For instance, defining a building as the composition of some materials, no intermediate layer would exist between material and building as the smallest and the largest composition layers. Instead by considering a building as a composition of walls, roofs, and windows, one intermediate layer would be defined as building elements. Similarly, French architect J. N. Huyot proposes the order of building composition as architectural elements (like shaft),
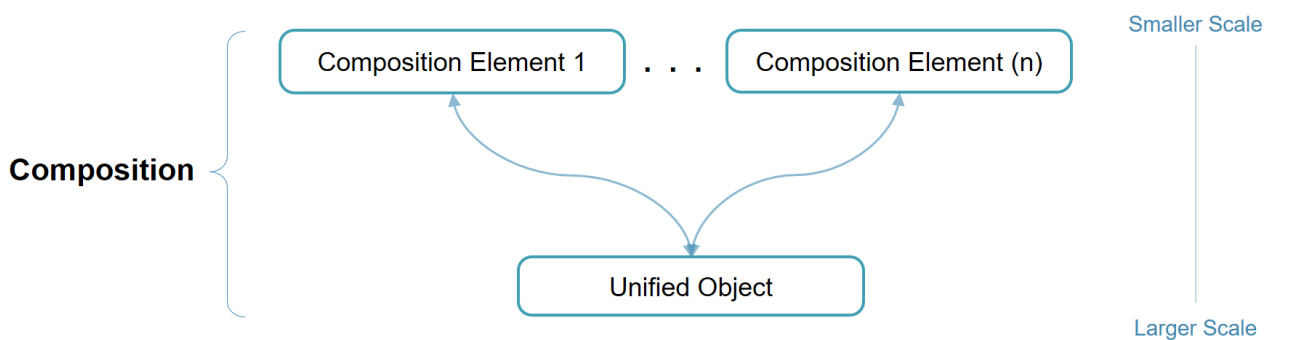

Composition Layer

Figure 1. Graphical composition definition \& distinguishing their composition layers via scale.
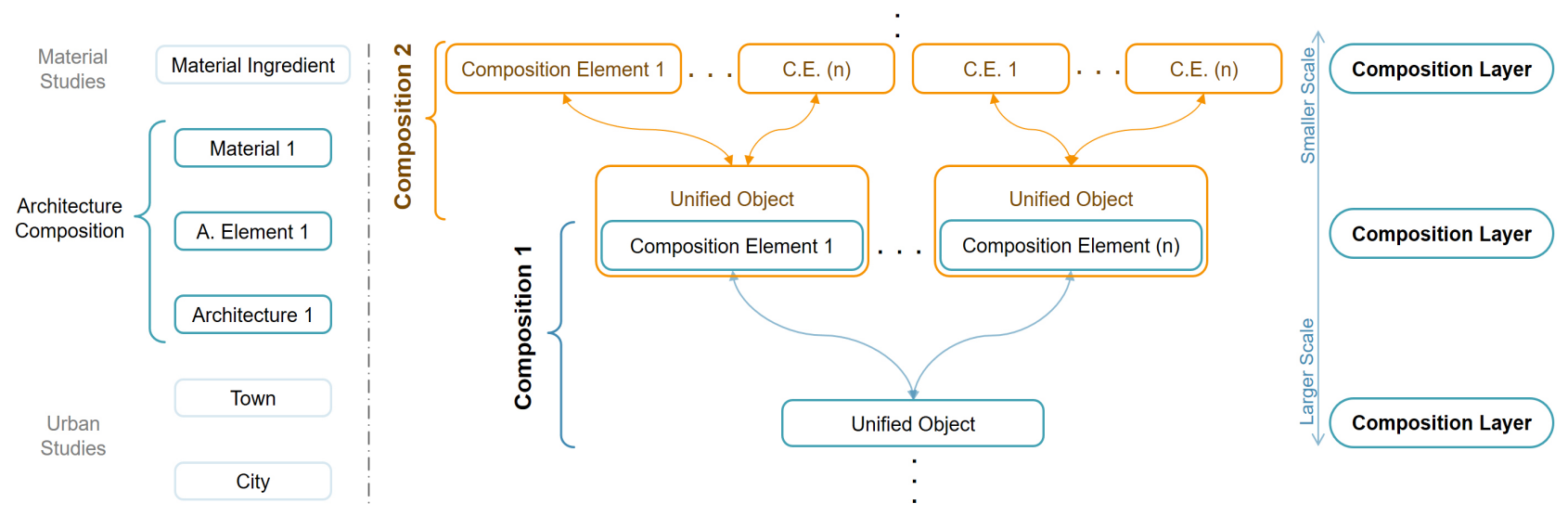

Figure 2. The chain of composition layers. 
architectural type (column with capital), a simple subject (like vestibule), and complex subjects (like building), regarded here as four composition layers ( $\mathrm{Lu}$ can, 2012: p. 88). Alternatively, the graph below illustrates 6 composition layers from the architectural material to building (Figure 3). Any composition can possess several numbers of composition layers. Worth mentioning, although the number of layers correlates in defining composition attributes, a higher number of layers do not necessarily lead to more comprehensive composition attributes. Rather, to have a well-organized list of attributes, instead of an exhaustive number of layers with little difference in scale, distinctive composition layers are required. Hence, the redundant intermediate layers should be discerned, as supposedly the three intermediate layers are eliminated in the graph below.

The considered scale-range and the number of intermediate layers are under the influence of various issues. The profound influence of material in the newly constructed buildings leaves no place to discern it as the smallest-scaled layer of composition. The ultimate unified object can be considered from part of a wall to a complex building. Since the introduced method aims to discover the attributes of real-world buildings, the number of intermediate layers is under the influence of various issues related to both observer and building properties, including observer's standpoint, its distance to the buildings, the accuracy of the perception, building details, overall building form, the number of elements, etc. Although various issues influence the number of the identified layers, the quantity of the layers is not a significant matter; instead, as far as the layers are distinctive and fully understandable, regardless of their quantity, they will lead to a proper list of composition attributes. The number of intermediate layers is adjustable to our needs, which will be clarified after having a general insight about the whole method.

\subsection{Composition Family and Their Properties}

After defining the proper composition layers, this step defines the components of each layer and their properties. Each distinctive component of a layer is called a family. For instance, if the materials of a building are brick, stone, and wood, the families of the material layer would be brick, stone, and wood. Noteworthy,
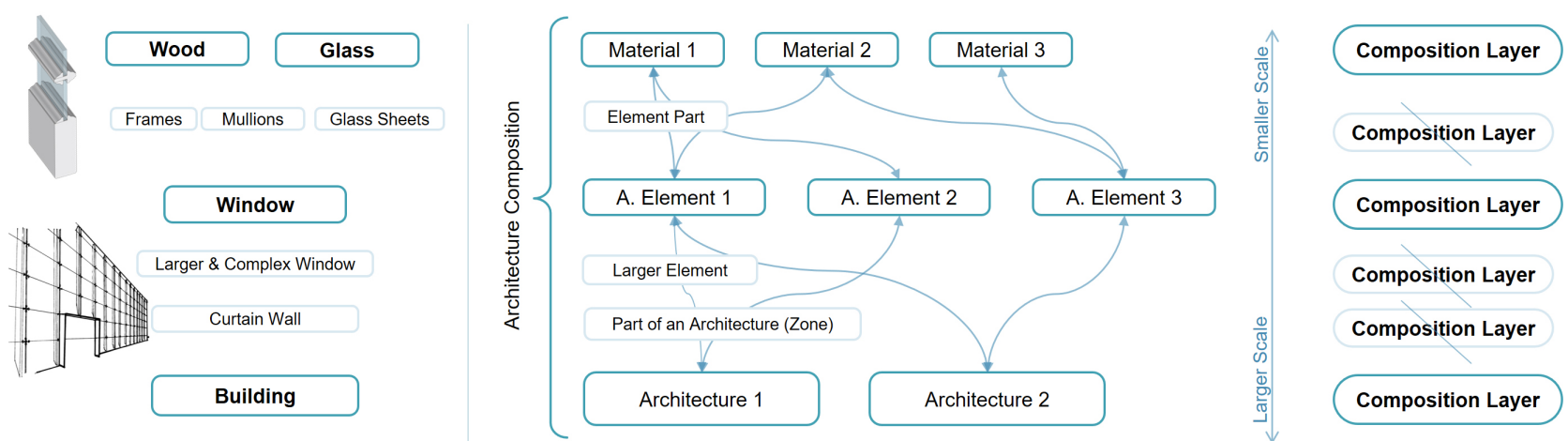

Figure 3. Various intermediate layers for a specific composition. 
each family must be independent of another family in the same layer; otherwise, the family belongs to another layer of composition, and the layer or family need revision. For instance, a combination of wood and brick is not a material family. Having the chain-like nature of composition in mind, each family of a layer is formed by the assemblage of some families in the smaller-scaled layer. For example, a distinctive wall as a family of element layer is formed by one or some families in material layer, like brick or wood. Therefore, the relationship between the families is gradually being revealed. Figure 4 samples a composition graph with 3 composition layers and 5 composition families, and a glance over the Figure 5 can facilitate its perception.

Following the procedure, each family has some properties. For instance, the properties of a material as a family member can be its color, texture, reflectivity and so forth. As graph 4 shows, the properties are written below each family. A proper list of the family properties can be obtained with the aid of theoretical discourses, personal experience, even comparing the families, as well as the software simulating realistic images. For example, 3ds Max lists various properties of a material in a user-friendly order; it can make an assistive list of properties such as quality, color, texture, pattern, pattern size, transparency, translucency, reflection, self-illumination, edge-properties, index of refraction, roughness, and so on. Since this study focuses on the visible composition attributes in building images, the very distinguishable properties need to be identified, rather than an extensive number of properties hard to specify. Until now, after defining the visible number of properties, a composition graph is prepared.

Arising from the composition graph, there are three main roots for the composition attributes: 1 ) The overview on the family (rectangular shapes in the graph), 2) the relationship between the families (lines in the graph), and 3) the family properties (the features under each family).

1) The families' overview leads to some composition attributes and their values. For example, the existence of just one family in the material layer will lead to the number of material (single material), as a composition attributes and its

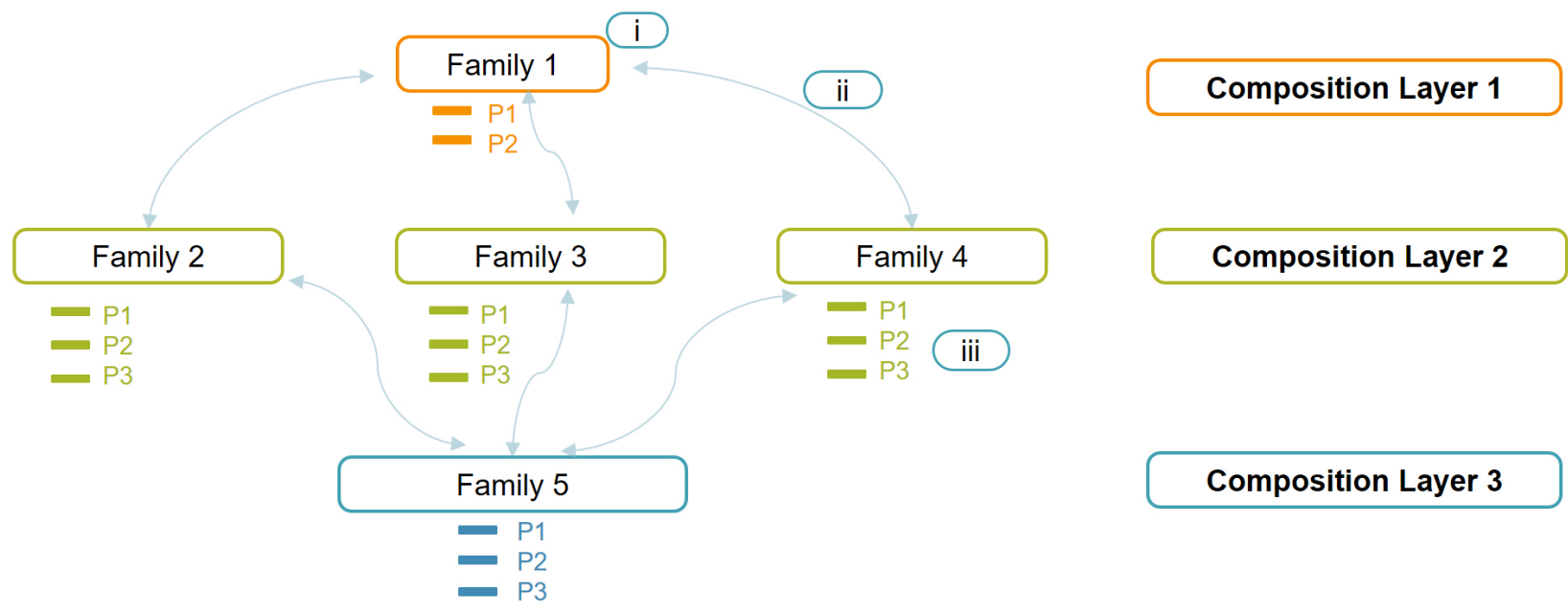

Figure 4. Sample of a composition graph (layers, families, family relationships, and family properties). 
value in parentheses; in case of existing five family members in the architectural element layer, then an attribute would be the number of elements (five or many). Worth mentioning, the number of the layers can reflect a composition attribute, compare graphs in Figure 5 and Figure 6.

2) The lines showing the relationship between the families reflect another set of composition attributes. For example, if all building elements had been formed by just one material, the number of material in each element (one) would be a composition attribute. The first two roots are the general overview of the graph.

3) In most cases, the family properties are the main source of composition attributes. Each property mostly reflects the value of the composition attributes. For instance, plain white as a property of a material, right-angle cube as a property of an element, and symmetry as a property of the unified object are considered as values for the following composition attributes: material color (with the value of white), material texture (no texture/plain), element geometry (right-angle cube), and symmetricity (symmetry).

Please consider, if there was just one family in a composition layer, then all of its properties can turn into some composition attributes. In case of existing more than one family in a composition layer, the relationship between them will lead to a meaningful list of composition attributes. For instance, if both black and white materials utilized in a building, relationship between their colors which is in-contrast would be the value of an attribute: material color (in-contrast). Noteworthy, although the method reflects various attributes, not all of them are significant and practically influential. The more detailed the graph is produced, the more number of attributes will be extracted. Finally, the most significant ones should be excerpted out of them. In case of focusing on some particular issues, the attributes need selection accordingly. Finally, the attributes needs refinements to have the most remarkable composition attributes. The method is applied on four samples selected in a way reflecting these considerations.

In case of applying the method for a set of buildings, the attributes and their values should be harmonized, since it make more sense to have a proper list covering them all. After gathering the significant composition attributes of each building, a set of attributes and their quantified values can be prepared, and each composition attributes and their values should be accordingly revised and adapted. For example, despite the existence of color spectrum, 6 values with a clear border can be defined, and the attributes can be valued accordingly, like (white, grey, black, light warm color, dark warm color, and cold color). That is, every color attributes has one of these 6 values, though it may add an ignorable "aboutness" to the values. This adaptation makes the measurement, comparison, finding similarities, and discovering the differences much easier; in brief, this harmonization makes the method more applicable for many further architectural investigations.

\section{Applying the Method}

The introduced method is applied on four buildings, to reflect the method in 


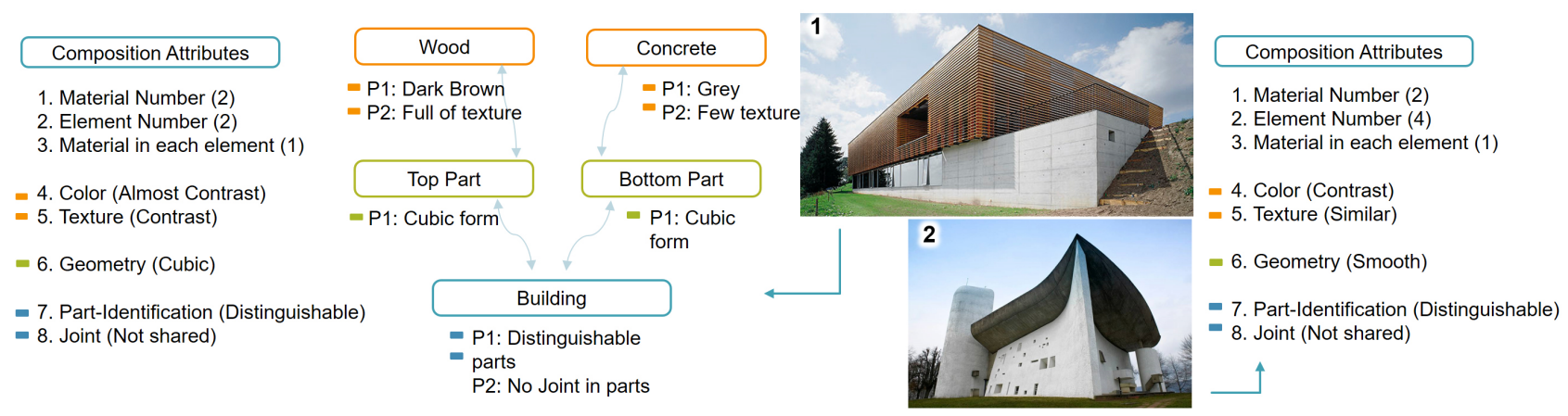

Figure 5. Composition attributes of samples 1 and 2.
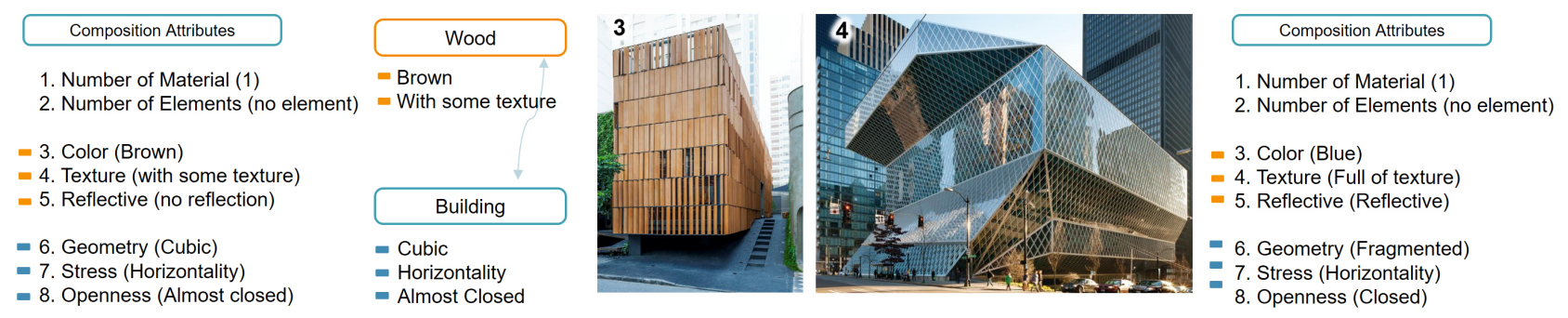

Figure 6. Composition attributes of samples 3 and 4.

practice: StamisolFa building, Ronchamp church, Bossa Restaurant, and Seattle Central Library, respectively designed by Serge Ferrari, Le Corbusier, Arco, and cooperation of Koolhaas and Prince-Ramus. The first two samples have very distinctive parts, which comprise one intermediate layer in their composition graph; they have three composition layers (Figure 5). The next two samples have a monolithic formal character; they have just 2 composition layers, without any intermediate layer (Figure 6). A glimpse over the Figure 5 and Figure 6 shows the difference between their general formal appearance and their composition layers. After defining their composition layers, the families of each layer are distinguished, and the composition graph is attained. Having a similar composition graph, for each pair of samples just one graph is illustrated. Lastly, for every single family, just 2 or 3 properties as examples are mentioned. Please consider, these samples are selected in a way to show the building attributes either with or without intermediate layer, to show different value of the same graphical model, and to confirm that the graph can reflect the differences of the visual attributes in a systematic manner.

Figure 5 reveals the composition graph and attributes of the first two samples. The graph overview, the families and their relationship, shows the existence of three attributes: both buildings have 2 materials with a few elements (Attributes $1 \& 2$ ) and in both buildings just one material forms each distinctive element (3). The family properties as another source of composition parameters reflect some other attributes. Regarding the existence of two families in a composition layer, the relationship between the properties echoes a more considerable attributes rather than each properties; both buildings use materials with contrast colors 
(4). In the first sample, one with a very dense texture and another has almost no texture; their material textures are in contrast (5). While in the second sample, both materials similarly have few textures (5). Having geometry as a property of both elements in the two buildings results in accepting geometry as a composition attribute with the value of cubic right-angular Pythagorean for the first and Smooth non-Pythagorean Sculptural-like for the second samples (6). There is no shared area/volume among the distinctive elements of the buildings; parts are distinguishable while there is no joint except their contours (7, and 8).

The next two buildings possess two composition layers, with just one family in each. Apart from the overall scheme of the graph reflecting some composition attributes, the whole family properties refer to the value of another set of composition attributes. As the graph displays, a single material forms each building (1). Having just two layers reflects lacking a composition element (2). Material quality can also introduce another composition attribute especially for single-material building (3), although the dominant material of a multi-material building can be mentioned as an attribute. The family properties reflect some other composition attributes like the material color (4) and material texture (5). In contrast to the third sample with ordinary absorbent wood, material reflection is an apparent property for the fourth sample material. Thus, material reflectivity is an attribute discovered by comparison, as it is mentioned as an assistive technique (6). Geometry is another composition attribute for both buildings, which is cubic and fragmented respectively (7). Based on the images, both buildings have no openings and possess slight stress on horizontality. Therefore, their stress (8) and openness (9) are mentioned as two composition attributes, although both buildings especially the library might be different in various photographs taken from a different stance or time.

Noticeable, some intermediate layers can be introduced for each building. For example, in the last sample, each surface of the fragmented form can be considered as an element. Then, its composition graph would have three composition layers; and the element properties can reflect some other attributes. For example, the shape and geometry of the plates could be among the composition attributes. In fact, the samples just illustrate the procedure and reveal the apparent composition attributes; rather than all the detailed attributes, which lastly require some refinements. It is important to know that the method is adjustable to the degree of accuracy we prefer to go through. If a special issue is the main concentration for us, the method would directly reflect those attributes; if we need a detailed composition attributes, the systematic method can be adaptable to the required precision; in case of concerning the main issues, the main visible and influential attributes would be acquired by applying the method with a few layers.

Furthermore, the parameters and their values reflect the composition attributes visible in its image, rather than building as an object. For instance, the color of a reflective material can differ in various weather conditions; the sample four would be more greyish in cloudy and bluish under sunny weathers. In addition, over the nighttime, the building appearance is entirely different, and their 
attributes alter accordingly. As another example, although the fourth building seems to have a solid skin covering the building, it is almost transparent from the inside. The images are the roots of the composition attributes, rather than the building as an object. Consequently, the introduced method would be perception-oriented method while applying to the real world buildings.

\section{The Most In-Common Composition Parameters}

Applying this method for a number of buildings can result in attaining a comprehensive list of the shared composition attributes, though it is not the aim of the paper; that is to say, although the introduced method aims to reflect the visual attributes of a building façade, it has this potential to disclose the shared ones among a set of building images. Accordingly, in a time-consuming process, over 200 building forms have been investigated via this procedure and a list of the shared composition attributes is prepared. There was no limitation over the building selections; the buildings are selected randomly, designed by several numbers of architects around the world. Since most of the recent building forms have no clear distinguishable parts, the shared composition attributes related to material and building layers as two ubiquitous composition layers. Finally, the shared attributes among the building are listed below (Table 1). Please consider, the prepared list is not a conclusive list of attribute, rather it shows that what attributes are more in-common among the buildings in general and reflects

Table 1. The most in-common composition attributes of the selected building images.

\begin{tabular}{|c|c|c|}
\hline \multicolumn{3}{|r|}{ Material-based Composition Attributes } \\
\hline Material Quality & 5 & Stone-Brick-Wood-Plaster/concrete-Aluminum \\
\hline Material Color & 6 & $\begin{array}{l}\text { White-Grey-Black-Light Warm Color-Dark Warm Color-Cold } \\
\text { Color }\end{array}$ \\
\hline Material Texture & 3 & Without Texture \& Pattern (T\&P) - With some T\&P-Full of T\&P \\
\hline Material Solidity & 3 & Solid-Almost Solid-Net shaped \\
\hline Material Reflectivity & 3 & Matt-Reflective-Very reflective \\
\hline Materials Quantity & 4 & $\begin{array}{l}\text { Single Material-2, } 3 \text { Different Material-2, } 3 \text { Contrast Material-Many } \\
\text { Materials }\end{array}$ \\
\hline \multicolumn{3}{|r|}{ Building-based Composition Attributes } \\
\hline Symmetricity & 3 & Symmetry-Partially Symmetry-Asymmetry \\
\hline Rhythm & 3 & Rhythmic-Partially Rhythmic-No Rhythm \\
\hline Pattern & 3 & Regular-Irregular-No Pattern \\
\hline Stress & 3 & Horizontality-Neutrality-Verticality \\
\hline Indentation & 3 & No Indent-Almost Indented-Fully Indented \\
\hline Complexity & 3 & Simple-Moderately Complicated-Complicated \\
\hline Decoration & 3 & No Decoration-Moderately Decorated-Fully Decorated \\
\hline Openness & 3 & Almost Open-Moderately Open-Almost Solid \\
\hline Geometry & 4 & Basic Geometry_Compound Geo._Fragmented Geo.-Smooth Geo. \\
\hline
\end{tabular}


that the method is applicable to extract the shared visual attributes among a set of building images. Although, this list can be a basis of future studies, we suggest to attaining an appropriate list of attributes that focuses more on the required characteristics of building in further formal exploration.

Apart from the attribute, the values are also limited to the most in-common easily-distinguishable features. For example, many architectural materials are being used in our time like stone, brick, wood, concrete, aluminum, plaster, mirror, glass, ceramic, Cor-Ten, copper, brass, porcelain tiles, creative Facade Panel, Cemintel Facade Panel, etc. Among them, just five commonly-used materials are mentioned. Also, the spectrum-like diverse values of some parameters are quantified into some analyzable and meaningful value groups, like the six mentioned values for the material color, and dividing the building openness into 3 classes. Finally, since the attributes are limited to the properties visible in the building images, the invisible, or undistinguishable ones are simply discerned like material durability, stiffness, index of refractions, glossiness and so on. The table below shows the shared composition attributes among the selected building.

\section{Conclusion}

This study introduces a systematic method for defining the composition attributes of a building. After preparing a composition graph and exploring their properties, the root of the attributes is attained. Accordingly, the composition attributes and their values are extracted in a more organized and objective manner. This system, by scrutinizing the composition parts and unified objects of a chain-like composition, reveals all the attributes of a composition in a more comprehensive manner.

This method has enough potential to reveal the attributes of various formal conditions. It can be applied on different composition scales, from wall to a complex building. Similarly, although the introduced method is applied on building images, it has potential to extract the composition attributes from a conceptual sketch to the extent of real-world perception of a building. Consequently, since the system arising from the definition of composition, it can potentially extract the composition parameters of anything the term composition covers. Interestingly, the system can be adjusted to our focal issues in our desired accuracy level. For instance, while the material is the main concern of the composition explorations, the attributes related to the first layer need consideration. While set of composition attribute is required, the number of layers, families, and their properties can reply the need, and introduce more-detailed composition attributes.

Noteworthy, applying this method on a set of buildings can reveal the shared and disparate composition attributes. By applying this method on a building group selected from a specific location or time, the shared attributes or values of the area or era will be exposed. If various buildings of an architect are analyzed, 
the architect's preferred attributes or formal values will be discovered. The method can reveal the personal, regional, geological, cultural, periodical composition attributes or values. Exploring various images of a building can reveal the shared or inconsistent attributes of its composition. Setting its vast application aside, this systematic method by its objective procedure explores visible composition parameters of buildings and prepares a list for further formal investigations.

\section{Acknowledgements}

This paper is prepared based upon the Ph.D. dissertation of Seyed Farhad Tayyebi, carried out under the supervision of Assoc. Prof. Dr. Yüksel Demir, at Istanbul Technical University, Turkey. The authors are immensely grateful to each member of the dissertation committee, Prof. Mehmet Nemutlu and Assoc. Prof. Dr. Can Karadoğan, for their extensive professional guidance over the dissertation progress.

\section{Conflicts of Interest}

The authors declare no conflicts of interest regarding the publication of this paper.

\section{References}

Albert, G. (2003). The Simple Secret to Better Painting: How to Immediately Improve Your Work with the Golden Rule of Design. Cincinnati, OH: North Light Books.

Aldrich, V. C. (1969). Design, Composition, and Symbol. The Journal of Aesthetics and Art Criticism, 27, 379-388. https://doi.org/10.2307/429424

Arnheim, R. (2009). The Dynamics of Architectural Form. Berkeley, CA: University of California Press.

Barnett, J. (2013). Urban Design, the Composition of Complexity. Journal of Urban Design, 18, 449-450. https://doi.org/10.1080/13574809.2013.800379

Brantingham, J. (2019). Art of Composing. http://www.artofcomposing.com

Breen, J. L. H. (2019). Patterns \& Variations: Designerly Explorations in Architectural Composition and Perception. Delft: Delft University of Technology.

Caniggia, G., \& Maffei, G. L. (2001). Architectural Composition and Building Typology: Interpreting Basic Building (Vol. 176). Siena: Alinea Editrice.

Clark, R. H., \& Pause, M. (2012). Precedents in Architecture: Analytic Diagrams, Formative Ideas, and Partis. Hoboken, NJ: John Wiley \& Sons.

Corbusier, L. (1954). The Modulor: A Harmonious Measure to the Human Scale Universally Applicable to Architecture and Mechanics. London: Faber and Faber.

Dews, P. (2003). The Painter's Workshop-Creative Composition \& Design. Cincinnati, $\mathrm{OH}: \mathrm{F}+\mathrm{W}$ Media, Inc.

Durand, J. N. L. (2000). Precis of the Lectures on Architecture: With Graphic Portion of the Lectures on Architecture (Britt, D., Trans.). Los Angeles, CA: Getty Publications.

Fallacara, G., Pantaleo, A., \& Scaltrito, G. (2019). Beech Wood for Architectural Design: Three Studies Case from an International Design Contest Terres de Hêtre BT. In F. Bianconi, \& M. Filippucci (Eds.), Digital Wood Design: Innovative Techniques of Representation in Architectural Design (pp. 1151-1181). Berlin: Springer. 
https://doi.org/10.1007/978-3-030-03676-8_47

Frutiger, A. (1989). Signs and Symbols: Their Design and Meaning (Bluhm, A., Trans.). New York: Watson-Guptill Publication.

Gargus, J. (1994). Ideas of Order: A Formal Approach to Architecture. Dubuque, IA: Kendall Hunt Publishing Company.

Grayson, C., \& Alberti, L. B. (1972). On Painting and On Sculpture: The Latin Texts of De Pictura and De Statua (p. 62). London: Phaidon Press.

Hanlon, D. (2009). Compositions in Architecture. Hoboken, NJ: Wiley.

Jiang, W., \& Qian, Z. (2019). Spatial Composition Patterns of Modern Medical-Elderly Care Buildings. Journal of Landscape Research, 11, 111-113.

Kasprisin, R. (2011). Urban Design: The Composition of Complexity. London: Routledge. https://books.google.com.tr/books?id=p31SXCRGEc0C https://doi.org/10.4324/9780203833766

Krier, R. (2010). Architectural Composition. Fellbach: Edition Axel Menges.

Kruger, C. (1991). Reviewed Work: Architectural Composition by Rob Krier. Journal of Architectural and Planning Research, 8, 266-268.

Langer, S. K. K. (1957). Problems of Art: Ten Philosophical Lectures. New York: Scribner.

Li, H. (2010). "Composition" and Regularisation of Architectural Production in Contemporary China. Frontiers of Architecture and Civil Engineering in China, 4, 465-473. https://doi.org/10.1007/s11709-010-0097-z

Lucan, J. (2012). Composition, Non-Composition: Architecture and Theory in the Nineteenth and Twentieth Centuries. Abingdon-on-Thames: Routledge.

Meiss, P. von. (2013). Elements of Architecture: From Form to Place. Abingdon-on-Thames: Routledge.

Schumacher, P. (2015). Design Parameters to Parametric Design. In M. Kanaani, \& D. Kopec (Eds.), The Routledge Companion for Architecture Design and Practice: Established and Emerging Trends (pp. 3-20). Abingdon-on-Thames: Taylor \& Francis.

Tokajuk, A. (2019). Multi-Family Buildings-Architectural Trends of the Last Three Decades in Poland. IOP Conference Series: Materials Science and Engineering, 471, 1-9. https://doi.org/10.1088/1757-899X/471/8/082022

Wright, F. L. (1928). In the Cause of Architecture: Composition as Method in Creation. In B. B. Pfeiffer (Ed.), Frank Lloyd Wright Collected Writings (pp. 259-260). New York: Random House Incorporated. 